\title{
How an Agent Can Detect and Use Synchrony Parameter of Its Own Interaction with a Human?
}

\author{
Ken Prepin ${ }^{1,2}$ and Philippe Gaussier ${ }^{2}$ \\ 1 LTCI lab, CNRS, Telecom-ParisTech \\ 46 rue Barrault \\ 75013, Paris, France \\ ken.prepin@telecom-paristech.fr \\ ${ }^{2}$ Neurocybernetic team, ETIS lab \\ Cergy-Pontoise University \\ 2, avenue Adolphe-Chauvin, Pontoise \\ 95302 Cergy-Pontoise, France
}

\begin{abstract}
Synchrony is claimed by psychology as a crucial parameter of any social interaction: to give to human a feeling of natural interaction, a feeling of agency [17, an agent must be able to synchronise with this human on appropriate time [29] [1] [15] [16] 27]. In the following experiment, we show that synchrony can be more than a state to reach during interaction, it can be a useable cue of the human's satisfaction and level of engagement concerning the ongoing interaction: the better is the interaction, the more synchronous with the agent is the human. We built an architecture that can acquire a human partner's level of synchrony and use this parameter to adapt the agent behavior. This architecture detects temporal relation [1] existing between the actions of the agent and the actions of the human. We used this detected level of synchrony as reinforcement for learning [6] the more constant the temporal relation between agent and human remains, the more positive is the reinforcement, conversely if the temporal relation varies above a threshold the reinforcement is negative. In a teaching task, this architecture enables naive humans to make the agent learn left-right associations just by the mean of intuitive interactions. The convergence of this learning reinforced by synchrony shows that synchrony conveys current information concerning human satisfaction and that we are able to extract and reuse this information to adapt the agent behavior appropriately.
\end{abstract}

Index Terms: Social interaction, intuitive social interaction, synchrony, active-listening, reinforcement learning.

\section{Introduction}

It is now clear that social interaction cannot be reduced to an exchange of explicit information. When an interaction takes place between two partners, it

A. Esposito et al. (Eds.): COST 2102 Int. Training School 2009, LNCS 5967, pp. 50-65, 2010.

(C) Springer-Verlag Berlin Heidelberg 2010 
comes with many non-verbal behaviours, such as imitations, perceptual crossing, facial expressions, and many para-verbal behaviours, such as phatics, backchannels or prosody. The first studies raising this issue of the form and the role of non-verbal and peri-verbal behaviours came from Condon et al. in 1966 and 1976 [5] 4. On one hand, these studies have described the non-verbal and periverbal behaviours cited just above, within dyads of persons engaged in discussion together. On the other hand, these studies have suggested that there are temporal correlations between the two behaviours of each dyad: micro analysis of videotaped discussions conduced Condon to define in 1976 the notions of autosynchrony (synchrony between the different modalities of an individual) and hetero-synchrony (synchrony between partners). Synchrony does not necessarily mean perfect co-occurence but constant temporal relation: just as described by Pikovsky et al., synchronisation can for instance be in anti-phase or with a phase shift [21].

The form of synchronisation between partners is even now investigated, by studying either behaviour [26] [25] [10] or cerebral activity [22] 28] 19] [20]. All these studies tend to show that when two persons interact together, they synchronise with each other or synchrony emerges between them. Synchrony is a dyadic parameter of the interaction between people, that means this parameter represents and accounts for the mutual coupling between them [12].

Synchrony does not only emerges from interaction, with this status of dyadic parameter, it can also be used by agents to modulate their interaction: it should participate to maintain contact between participants, facilitate verbal exchange and may also convey information. Psychological studies of dyadic interactions between mother and infant showed that very early in life (since two months and certainly earlier) synchrony between partners is a necessary condition to enable interaction: the infant stops interacting and imitating her mother when the mother stops being synchronous with her, all other parameters staying equal 29] 11 15] 16, 18, 27. To explain this early effect of synchrony, Gergely and Watson postulate an innate Contingency Detection Module (CDM) 7] 8]. This detection of synchrony will enable the infant to detect the reactivity of her mother; when her mother is contingent, the infant is able to detect relations between her own actions and the actions she perceives from her mother: the action of her mother become a biofeedback of the infant's own actions. To detect the synchrony of the other is not only to detect the reactivity of the other, it is also to detect her/his engagement within the ongoing interaction and moreover it is also to detect her/his agency [17. In that way, synchrony has been shown to be a premise of the interaction: in Nadel's Still Face experiment 13], the experimenter faces an autistic child which first ignores her. She then forces the synchrony with the child by imitating him, and the child enters in interaction with the experimenter. Child and experimenter finish taking turns and imitating each other. In that case forced synchrony made the child with autism detect the experimenter as a social partner.

This literature contributes to show that some of the signals created by the social interaction, such as synchrony between partners' behaviours, are signals 
which both enable to bootstrap, regulate and maintain social interaction and also signals which enable to develop a sense of agency and which contain information concerning the ongoing interaction.

How can we build a human-robot interaction which could take advantage of the signals emerging from this interaction so as to self-regulate? And how can we evaluate if the robot involved within this interaction does extract and use these signals?

A dyadic parameter of the interaction such as synchrony is a global parameter of the dyad, which makes sense only when we speak about several systems, but which is also perceivable by each individu of the dyad. That is the interesting point of this parameter, it carries dyadic information, concerning the quality of the ongoing interaction, and at the same time it can be retrieved by each partner of the interaction.

Andry et al. 1] used the rhythm of human actions on a keyboard as a reinforcement signal for learning. They assumed that when a human is satisfied with the computer answers, her/his actions are regular and will give positive reinforcement, and conversly, a break of the rhythm will produce an error signal. In the present paper, we also propose to learn some simple associations being only guided by an implicit reinforcement. In our case, the human and the agent will interact, not through a keyboard and a screen, but in the real world, through action and mutual perception: the agent will be a robot. The reinforcement will not be given by the individual parameter of actions rhythm (of either a human [2] or an agent [1) but by the dyadic parameter of synchrony, which links the behaviour of both partners and which should be naturally modulated by their mutual engagement. The convergence of the learning reinforced by synchrony will show two things: First, that the dyadic parameters of the interaction emerging from the coupling between agents carry information available for each individual taking part within the interaction. Second, that these signals can be obtained and re-used by the robot and enable it to benefit from the interaction, for instance by learning a rule linked to the partner.

We have built a robot, ADRIANA (ADaptable Robotics for Interaction ANAlysis, 23]), capable to get the information of synchrony of its interaction with a human. We present first the principle of the experiment and of the associated architecture. Then we present in detail the architecture, from the synchrony detection to the use of this synchrony as a reinforcement signal. In section four we present our results on live experimentations with naive human subjects: seven naive subjects enable to improve the robot and architecture kinematics, and then three naive subjects were presented to the fully functionning robot to test its ability to detect and use synchrony. Finally we discuss these results which annonce a step toward intuitive interaction between human and robot, where human has not any knowledge of the robot functionning and only interacts with the implicit rules of every dyad in interaction. 


\section{How to Extract Relevant Cues of Ongoing Interaction and Proove That?}

The aim of this robotic architecture and of its associated experiment is dual. On one hand the architecture should enable the robot to extract synchrony information during an interaction. On the other hand the experiment should prove that the extracted synchrony is relevant for social interactions, that means that synchrony is naturally modulated by human during an interaction.

To prove that, we will use the extracted synchrony to reinforce a learning: if the learning converges, then the synchrony we used is relevant, if the learning does not converge, then the signal we extracted is not appropriate.

\subsection{Experimental Procedure}

Each naive human subject faces the robot ADRIANA. ADRIANA is equiped with two arms with one degree of freedom and a camera (see fig.1). When the human subject, raises or pulls down one of her arms, the robot, respectively, raises or pulls down one of its arms, randomly the left or the right: the robot imitates the up or down position but not the left and right.
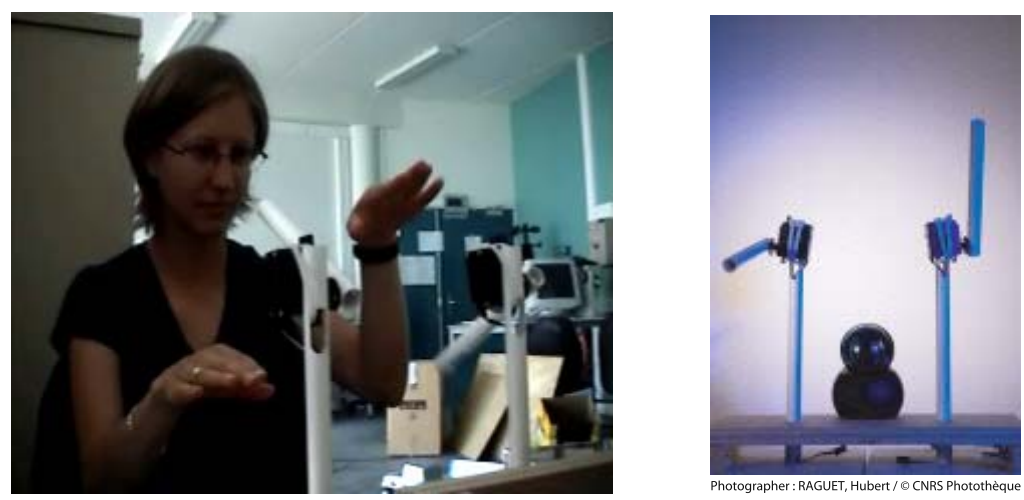

Fig. 1. A naive human subject faces the robot, ADRIANA 23. (right picture), which is equiped with two arms with one degree of freedom and a camera. When the human raises or pulls down one of her arms, the robot respectively raises or pulls down one of its arms. The subject is asked to make the robot learn to imitate "in mirror" (to move the arm on the same side as the one moved by the human).

The instruction given to the naive subject is to "make the robot learn to move the arm which is on the same side as the one you move (in mirror)". The subject does not know how the robot learns and does not know how she/he can influence it. The only way is to try to interact with the robot. If the learning converges in these conditions, that will prove that the cues detected by the architecture are relevant cues of human communication: they are modulated 
by the human naturally, without having been asked to do so; these intuitive modulations carry information concerning the ongoing interaction, they have enable the convergence of learning. If, furthermore, these cues detected by the architecture are the synchrony variations, that will also prove that synchrony is a parameter naturally modulated depending on the ongoing interaction.

\subsection{Architecture Principle}

The idea of this architecture is to retrieve the information of synchrony between partners and to use it to reinforce associations between left/right visual field and left/right arms. We assume that synchrony is an indice of partner satisfaction concerning the ongoing interaction.

Synchrony is a dyadic parameter which characterises the reciprocal engagement of two partners. From the whole two partners system (the dyad), synchrony is accessible measuring the temporal relation existing between the actions of one partner and the ones of the other. But from the individual point of view of each partner, this information is also accessible, by comparing the time of her/his own action (ideally using proprioception) and the time of the successive activation within her/his visual field, generated by answer of the other partner.

To enable ADRIANA to compare its actions to the human's actions and to detect synchrony, there are two ways in the architecture, one from its perception and another from its action. If the delay between activation of the action way and activation of the perception way remains constant, that means that human and robot are synchronised: the interaction goes as human expects. In that case the reinforcement of previously used associations will be positive. If the delay predicted between perception and action varies above a threeshold, that mean that there is no synchrony between human and robot: the interaction is temporarily disrupted, accounting for unsatisfied human's expectations. In that case, the reinforcement of previously used associations will be negative.

Finally, the convergence of learning will show that the reinforcement signal (the synchrony extracted) is correctly built by the architecture and that the synchrony modulated by the human interacting with the robot is an indice of the satisfaction of human's expectations.

\section{Detailed Architecture}

Figure 2 shows the full architecture, from visual detection of movement to motor commands sent to the arms. The pathway between perception and action is modulated by the detection of synchrony. In the remaining of this section, we detail the different part of this architecture.

\subsection{Synchrony Detection: The Delay Prediction}

To detect synchrony with the interaction partner, the architecture predicts the delay between its own actions and the detected actions of the partner. To predict 


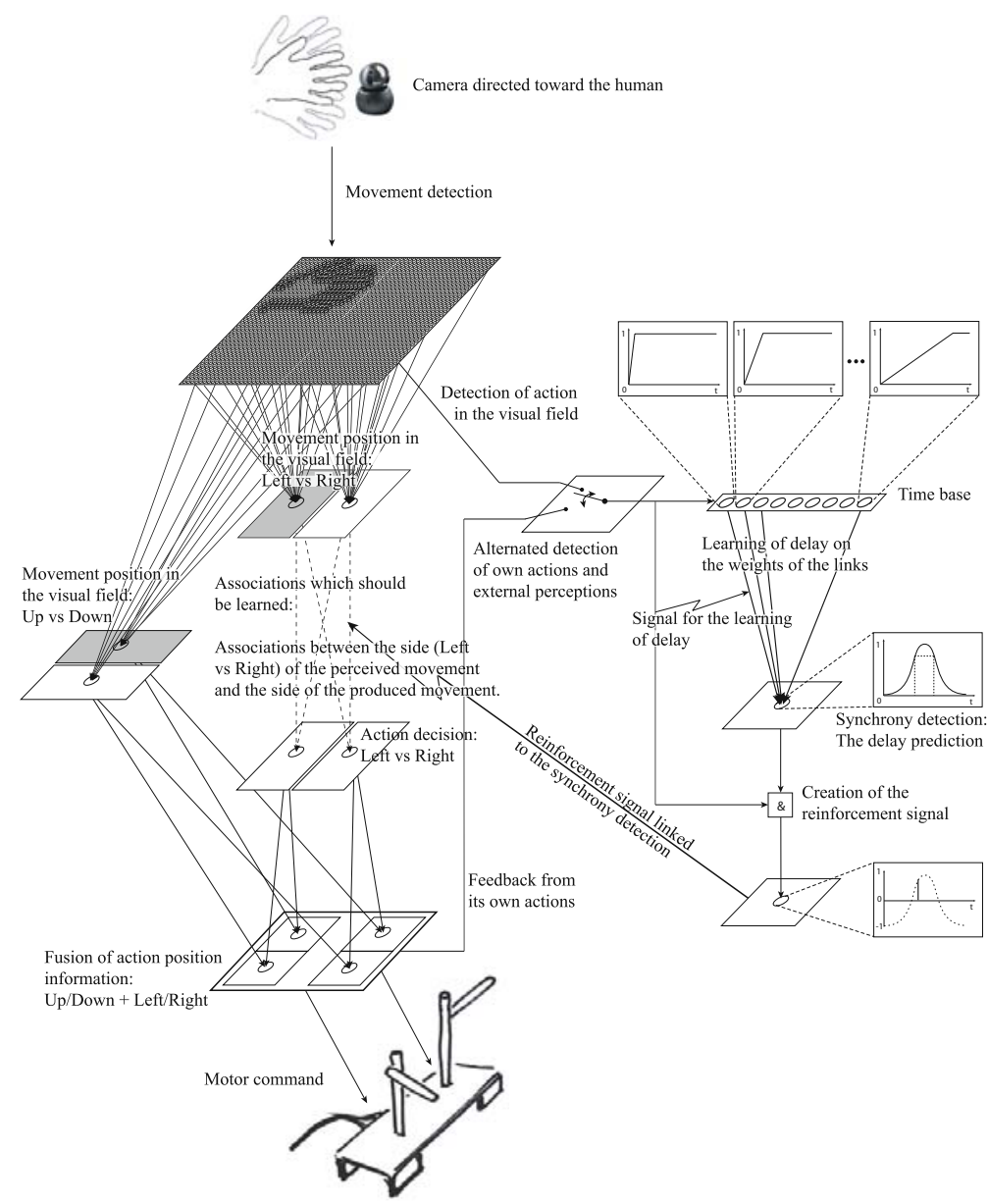

Fig. 2. ADRIANA's architecture. This architecture enable the robot, when it is interacting with a human, to detect the social signal naturally modulated by the human, here the synchrony, and to use this signal to reinforce a learning.

delay between action and perception we used a modified version of the architecture proposed by Andry et al. in 2001 which enables one to learn on the fly complex temporal sequences [1]. The original architecture enables a one shot learning of a temporal sequence of signals thanks to two formal neurons groups, connected together by modifiable links.

The first group of neurons is a time base1: every neuron is activated by a unique entry but each one has a different activation dynamic, from very quick

${ }^{1}$ See the works of Grossberg and Merryl 1996, and Banquet et al 1997, for a more neurobiologically plausible implementation of neurons with a time spectrum activity 9] [3]. 
to very slow. The input activates every neuron simultaneously, for instance at time $t_{0}$, each neuron has an activation dynamic $A c t_{k}(t)$ which depends on the neuron position $k$ in the group: $A c t_{k}(t)=\frac{n}{k} \alpha\left(t-t_{0}\right)$ (where $n$ is the number of neurons in the group and $\alpha$ a constant coefficient, see graphics of the upper part of fig(3). As soon as this group is activated by an input, the pattern of activations of its neurons represent the course of time since the input. It is this pattern of activations which will be learned and which will enable prediction of delay. To enable the architecture to predict delay between its own actions and the actions of the other, we have added a switch between signal coming from perceptive pathway and motor pathway. The time base is activated by the signal coming from the motor pathway and the pattern of the time base activations is learned when a signal coming from the perceptive pathway occurs.

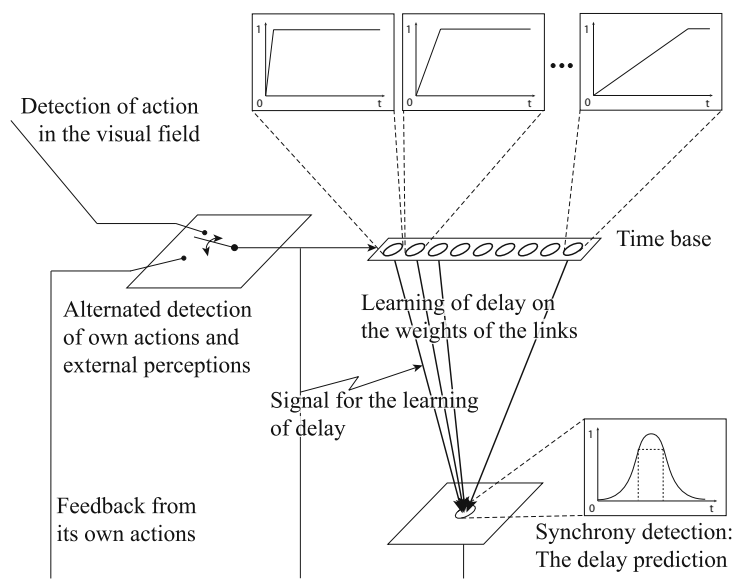

Fig. 3. Synchrony detection. This part of the architecture is dedicated to the prediction of the delay between the robot's own actions and human's actions: i.e. synchrony between human and robot. A first group of neurons, the time base, "measures" the course of time as soon as the robot produces an action. Links toward a second group of neurons, the delay prediction, have their weights modified when an action of the human is detected. The weights store the delay measured by the time base.

The second group is the delay prediction. In our case it is a group of one neuron as we have only one delay to predict. This neuron is connected to every neuron of the time base group (one to all connection). Andry et al. 1 propose a one shot learning of the delay $d_{i}$ between two successives events $e v_{i}$ and $e v_{i+1}$ of a sequence. To do so, after $e v_{i}$, when the next event $e v_{i+1}$ occurs, the pattern of activations of the time base neurons is stored in the weights $W_{k}$ of the one to all links between the two groups: $W_{k}(t)=A c t_{k}(t)$, and the time base is reset. We have modified this one shot learning rule to enable a learning of the mean value of the delay according to a Kohonen like learning rule: $W_{k}(t+1)=$ $W_{k}(t)+\mu \times\left(A c t_{k}(t+1)-W_{k}(t)\right)$, where $\mu$ is the learning speed $\left(\mu=\frac{1}{3}\right.$ in 
our case, if $\mu=1$ it becomes a one shot learning). It is necessary to predict a mean delay since this mean delay is representative of the ongoing interaction which takes place between human and robot: when the interaction is satisfying, the human comes back to this "phase shift" when she/he aswers to the robot's actions. When the time base is activated once again, by a new motor command of the robot, the neuron of the second group is activated depending on the learned weight, according to the following equation: $\frac{1}{n} \sum_{k=1}^{n} e^{-\frac{1}{2 \delta^{2}}\left(A c t_{k}-W_{k}\right)^{2}}$ where $\delta$ is a coefficient controlling the width of the Gaussian $e^{-\frac{1}{2 \delta^{2}}(x)^{2}}$ (in our case $\delta=0.2$, see next section). The prediction of delay is a sum of gaussians: one gaussian for each neuron of the time base, centered on the predicted delay, when $A_{c} t_{k}=W_{k}$. This signal, also a gaussian centered on the predicted delay, will enable the architecture to built a reinforcement signal (see graph in the lowest right part of fig 3 .

\subsection{Reinforcement Signal: Synchrony}

The prediction of the delay between action and perception is used by the architecture to compute the "level of synchrony" of the human-robot dyad. This level of synchrony is the error between the predicted delay and the real delay: If the predicted delay is close to the real delay, there is constant timing between both partners of the dyad, there is synchrony within the dyad and the interaction might be satisfactory for both partners (in our case for the human, the robot is always engaged in the interaction). If the predicted delay is far from the real delay, timing between partners of the dyad has varied; there is a synchrony break within the dyad; the interaction may have been disrupted by some event (in our case, by the robot which does not satisfy human expectations).

Synchrony between partners of an interaction should account for the quality of the interaction. In our experiment, we use this synchrony as reinforcement signal, assuming that the more satisfied will be the human expectation, the more synchronous the human will be with the robot: the more predictible the delay between robot's actions and human's actions will be.

To build this reinforcement signal, the robot can use two things: on one hand it has the delay it is currently predicting, a gaussian centered on the learned delay (see section 3.1), and on the other hand it has the real current delay, instantaneous signal issuing from the perception of an action in the visual field (see fig 4).

The reinforcement signal Renf is the value of the gaussian when an action is perceived in the visual field, i.e. when a signal comes from the movement detection. This reinforcement signal is computed at the level of the \& operator in figure 4 .

This reinforcement signal which varies between 0 and 1 will then be projected between -1 and 1 to enable positive or negative reinforcements (see the lower graph of figure 4). The width $\delta$ of the gaussian must be chosen carefully: it must be large enough to tolerate small variations in the synchrony between human and robot, but also narrow enough to enable detection of synchrony breaks associated to human subject unsatisfaction. We have chosen $\delta=0.2$ 


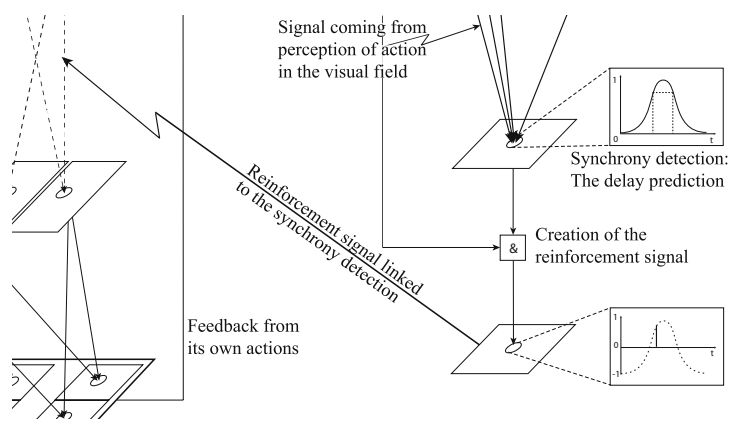

Fig. 4. From synchrony to reinforcement signal. The reinforcement signal is built depending on the quality of the interaction, i.e. depending if the expectations of the human subject are satisfied or not. The more satisfied is the human, the more regular will be her/his productions after the robot actions, thus, the more accurate the predicted delay will be. When the human's action is detected, the current value of the predicted delay, a gaussian centered on the predicted delay, is taken. This value is between 0 and 1, maximum when the prediction is exact and which lower when the real delay moves away (advanced or delayed) from the prediction.

which corresponds to a tolerance of about $0.4 \mathrm{sec}$ in the delay prediction, before sending a negative reinforcement.

Finally, better is the predicted delay compared to the real delay, better is the reinforcement signal, thus better is the reinforcement, better is the synchrony between human and robot behaviours.

\subsection{Learning Based on a Delayed Reinforcement}

In order to test the relevance of this "level of synchrony" we used it as a reinforcement signal for the learning of associations: the relevance of this computing will be validated if the "level of synchrony" computed by the architecture enables learning to converge. The learning modulates associations between the side of perception of the robot (left or right visual hemi-field according to the arm moved by the human) and the side of action of the robot (left or right arm of the robot).

The reinforcement signal (the level of synchrony) is generated by the human (who ignores that) and we do not know a priori when this signal will vary after an error of the robot. That raises two issues: first the reinforcement signal occurs later than the trials it concerns; second the perceptions-actions associations aimed by the reinforcement signal are not specified. The PCR (Probabilistic Conditioning Rule) of Gaussier and Revel et al. 6 24 solve the first issue: it enables associations between groups of formal neurons to be modified according to a reinforcement signal, even if the reinforcement occurs later than the trials it concerns. The second issue remains since the original PCR was built for a reinforcement signal aiming at a well defined set of associations: the associations used since the previous reinforcement. By contrast, the reinforcement signal linked to 
the level of synchrony is computed at every trial and we do not know exactly how many associations it concerns. To solve this second issue, we assume that humans naturally make synchrony vary according to her/his satisfaction and we assume that this synchrony modification detected by the architecture (see section 3.2) concerns the actions performed by the robot an a time window of length $\tau(\tau=3$ seems a good length for this window, see section 4 4 . We modified the PCR so that the reinforcement signal at time $t$ concerns the associations used during the time window $[t-\tau, t]$.

The learning rule is a local rule, i.e. it applies to one link between an input neuron and an output neuron and depends only on the activation of these two neurons and on its own weight. Given a link $I_{i} \rightarrow O_{j}$, at each time step, the activation rate $A c t E_{i}$ of its input $E_{i}$ is updated. $A c t E_{i}$ is the percentage of activation times during a window of $\tau$ time steps: $A c t E_{i}(t+1)=\frac{\tau \times A c t E_{i}(t)+5 \times E_{i}(t)}{\tau+5}$ where $E_{i}=0$ or 1 .

When a reinforcement signal is produced by the synchrony detection (section 3.2), Renf $\neq 0$, two additional variables are updated:

- First the confidence $C_{i j}$ in the weight of the link. It depends for one part on the reinforcement signal (common to every link) and for another part on the impact of this specific link on the reinforcement signal, i.e. the activation rate $A c t E_{i}$ of the entry of the link:

$C_{i j}(t+1)=C_{i j}(t)+\alpha \times \operatorname{Renf}(t) \times \operatorname{Act} E_{i}(t)$ where $\alpha=0.5$

To compute the variation of the confidence in the weight of the link, the reinforcement signal is first projected from $[0 ; 1]$ to $[-1 ; 1]$ and then multiplied by the activation rate $A c t E_{i}$ of the entry and a coefficient $\alpha$. This variation is added to the previous confidence $C_{i j}$.

- After that, the weight $W_{i j}$ of the link is updated depending on $C_{i j}$. The confidence $C_{i} j$ is used as a probability to maintain the weight value unchanged: If a random draw is greater than or equal to $C_{i j}$, then $W_{i j}(t+1)=1-W_{i j}(t)$ and $C_{i j}(t+1)=1-C_{i j}(t)$.

Else, the weight is unchanged.

When the random draw is greater than the confidence $C_{i j}$ in the weight, the weight is drastically modified (symetrically to 0.5 ), and thus the confidence in this new weight is also modified (symetrically to 0.5 ).

These different parts of the architecture, when put together and correctly parametrised, enable the robot to use the synchrony of its interaction with a human as a reinforcement signal.

\section{Results}

ADRIANA equiped with two arms, a camera and the previously described architecture, has been first parametrised on seven naive subjects and then tested on three other naive subjects.

The first seven subjects enabled us to improve the timing of the different parts of the architecture of the robot: 
- Our system does not modulate its rhythm of interaction in live, the arms velocity and the speed of answers to visual stimulation, have been adjusted along these first experiments: To enable human interaction rhythm to be intuitive for the subject, the human needs to feel reciprocal interaction. The reaction of the robot to human's movements must be systematic and almost instantaneous to be trusted to be imitation. It must also be predictible by the human, not in its form but in the fact that particular movement of the human will systematically lead to an answer of the robot. The human must be sure that both she/he is influencing the robot and she/he has the attention of the robot. Otherwise, the human is rapidly discouraged.

- The kinematics of the robot's movement must fit the kinematics perceivable by the robot: the human adapts the kinematics of his actions to the robot kinematics, systematically and with no instruction. It is one facet of the expertise of human in social interaction. For our robot, a good compromise between speed and detection is full movements (raising or pulling down an arm) which last around 1 second: human-robot reciprocal answers are fluid, human actions are systematically detected by the robot, and time delay from an action to the next (between 1 and 2.5 seconds) are also correctly predicted by the robot.

- The kinematics of the robot, kinematics of human induced by the robot and kinematics perceivable by the robot taken as a whole, must be what is intuitively modulated by human when socially interacting, i.e. the interindividual synchrony. In our case, for actions lasting 1 second, the mean delay of human reactions to the robot actions is $1.5 \mathrm{sec}$, between $1.4 \mathrm{sec}$ and $1.7 \mathrm{sec}$ when the interaction is going well, and modified by more than $0.5 \mathrm{sec}$ when perturbations such as robot's mistakes occur (the width of gaussian $\delta=0.2$ which corresponds to a tolerance of $0.4 \mathrm{sec}$ ).

- The reinforcement signal comes around 4 seconds (equivalent to about 3 actions) after the corresponding actions of the robot: the reinforcement must concern a time window with a length of at least 3 steps $(\tau=3)$.

- These points contribute to make the robot's production of action to enable synchrony to emerge between human and robot.

The last three subjects have been presented to the whole experimental protocol (without anymore adaptation of the robotic setup). They could interact naturally and robustly with the robot and made the learning converge. A second task was added due to the quick and good results of the first teaching task:

The first instruction given to the subject was to make the robot learn to imitate "in mirror" (for an example see http://ken.prepin.free.fr/spip.php?article20, first video "The robot which uses natural cues of social interaction. Part 1").

Then, when the robot did not make anymore mistake (learning has converged and the subject has the feeling of stabilised behaviour), subject have been asked to make the robot imitate "on the opposite side". There was no break in the experiment, the robot had to unlearn previous association and learn new ones, on the fly (for an example see http://ken.prepin.free.fr/spip.php?article20, second video "The robot which uses natural cues of social interaction. Part 2"). 
The results obtained in these three experiments are similar (see fig 5 for an example):

- Learning converged: in our three experiments, an average of 10 reinforcement signals was necessary to enable learning to converge, 30 signals to unlearn and relearn associations. This learning convergence was faster than learning by chance: let $N$ be the number of possible associations (4 in our case: two possibilities for each of the two possible inputs), let $\tau$ be the size of
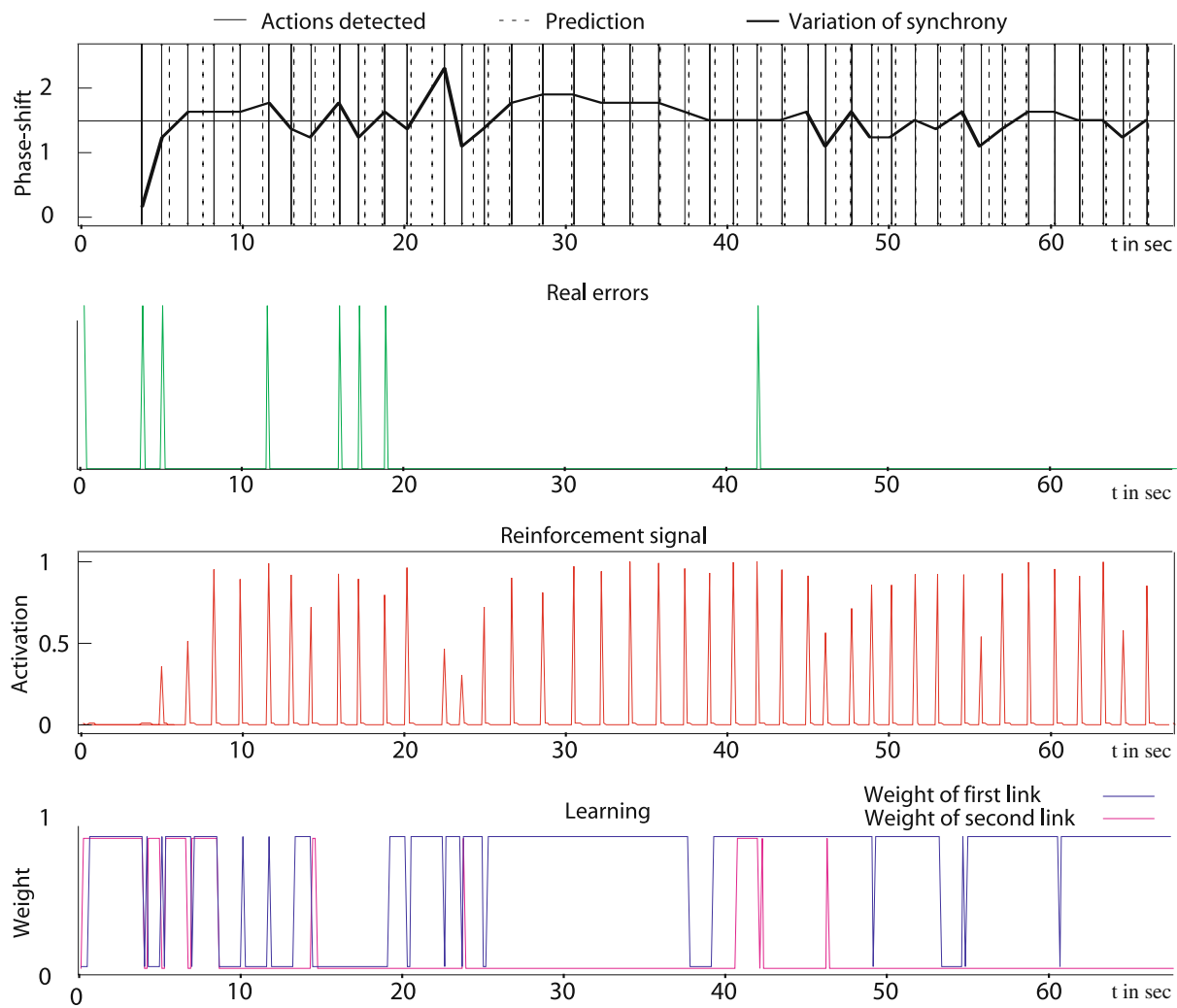

Fig. 5. Results obtained with one subject. The first graph represents together the real (continuous line) and predicted (dotted line) actions of the human and the associated phase-shift between robot and human (bold line). The second curve represents the real mistakes of the robot (according to the instructions: "make the robot imitate in mirror"). The third curve is the reinforcement signal computed using synchrony information: a negative reinforcement is lower than 0.5 and a positive reinforcement is higher than 0.5. This reinforcement depends on the accuracy of the predicted delay compared to the real one. The fourth graph is the evolution of the weight of the links between perception and action. It enable to see if these weights stabilise and if they are opposite (as needed). Let us notice that learning stabilises and moreover that reinforcement signal is clearly linked to the real mistakes of the robot. 
the time window concerned by the reinforcement signal (3 in our case: the reinforcement signal depends on the three previous detections of synchrony) and let us assume that the reinforcement can be either positive or negative. In the same conditions (delayed positive or negative reinforcement), a random reinforcement would have $\frac{1}{N} \times \frac{1}{\tau} \times \frac{1}{2}=\frac{1}{24}$ chances to be correct.

- Learning converged in reasonnable time: learning converged in less than half a minute, unlearning and relearning took 1 min 30 sec.

- At the end of the experiment, while "debriefing", the three subjects spontaneously declared to have enjoyed the experiment.

- The three subjects spontaneously declared they had the feeling of having influenced the robot.

- The three subjects declared they were satisfied with the experiment and were ready for a new one.

\section{Discussion}

This study leads to two main results.

The most important is that the information of synchrony, naturally given by the naive user, brings relevant sense. Synchrony is not only a phenomenon accompanying every social interaction, it is a parameter which carries information concerning the ongoing interaction. Our results participate to show that this dyadic parameter can be detected and used by each agent involved in the interaction so as to get information on this interaction. In our case, synchrony has conveid information relevant for learning showing that it was directly linked to the satisfaction of naive human subjects' expectations regarding the robot's actions.

The second result is that this architecture enables us to extract and re-use this information. The comparison between actions of the agent and perception is a mean to detect synchrony. The proposed experiment design makes this detection easy: the very simple robot, ADRIANA, and its basic movements lead the human to produce action easily detected by our system, i.e. bounded in time and extractable only by movement detection. The associated learning by a delayed reinforcement enabled us to benefit from this synchrony detection even if we did not known a priori its exact relevance and precision over time. This architecture should be an inspiring way to improve HMIs (Human Machine Interfaces) by allowing an intuitive on line learning with naive users.

These two intwinned results, support the idea that an agent's ability to interact with a human and to perceive the relevant signals of this interaction, is not only a matter of technical complexity of its detection system. Both the agent and its behaviours influence the way and the means used by human during interaction: with a simple robot which only raises or pulls down its arms with a specific kinematics, human will interact just raising and pulling down its arms with similar kinematics, easily detectable by the robot. Finally, the agent's own productions influence its possibility of detection and perception.

To investigate synchrony more in details the idea would be to enable the architecture to modify its dynamics depending either on internal states (or motivations) 
or on the detected synchrony (with for instance synchronisation mechanisms such as dynamical coupling [23]). On one hand, the agent may be able to test the engagement of the human: the agent can modify its production dynamics and measure the subsequent effect of this modification on the human partner; if the human stays synchronous eventhough dynamics have changed, it means she/he is engaged within the interaction, otherwise it means either she/he does not care about the interaction or she/he has expectations not satisfied. On the other hand, the agent may be able to show either its engagement or its un-satisfaction by respectively synchronising or un-synchronising with the human. Such abilities of the social agent would fit the experimental psychology claim that social agents test the engagement of their partner by modifying their production and controlling the reaction of the partner.

Finally, during the interactions enabled by our architecture, knowledge has been exchanged between human and robot. But the form of this exchange contrasts with typical views: Here the knowledge is not directly contained in the transmitted information as it can be when language is used or when a robot imitates a human. Here the robot extracts information from the course of the interaction itself: what is taken into account by the robot is not the transmitted information but the way the interaction evolves through time. The only mean to make this robot learn something is not to show it, to repeat it, to correct it, the only mean to make this robot learn something is to interact with it, to be trully engaged in interaction with it.

\section{Acknowledgements}

This work has been mainly financed by a research grant from the DGA and lately supported by the NoE SSPNet (Social Signal Processing Network) European project.

\section{References}

1. Andry, P., Gaussier, P., Moga, S., Banquet, J.P., Nadel, J.: Learning and Communication in Imitation: An Autonomous Robot Perspective. IEEE transactions on Systems, Man and Cybernetics, Part A 31(5), 431-444 (2001)

2. Andry, P., Moga, S., Gaussier, P., Revel, A., Nadel, J.: Imitation: learning and communication. In: The Sixth International Conference on Simulation for Adaptive Behavior SAB 2000, Paris, pp. 353-362. MIT Press, Cambridge (2000)

3. Banquet, J.P., Gaussier, P., Dreher, J.C., Joulain, C., Revel, A., Günther, W.: Space-time, order, and hierarchy in fronto-hippocampal system: A neural basis of personality. In: Matthews, G. (ed.) Advances in Psychology, Amsterdam, vol. 124, pp. 123-189. North-Holland, Amsterdam (1997)

4. Condon, W.S.: An analysis of behavioral organisation. Sign Language Studies 13, 285-318 (1976)

5. Condon, W.S., Ogston, W.D.: Sound film analysis of normal and pathological behavior patterns. Journal of Nervous and Mental Disease 143, 338-347 (1966)

6. Gaussier, P., Revel, A., Joulain, C., Zrehen, S.: Living in a partially structured environment: How to bypass the limitation of classical reinforcement techniques. Robotics and Autonomous Systems 20, 225-250 (1997) 
7. Gergely, G., Watson, J.: The social biofeedback model of parental affect-mirroring. International Journal of Psycho-Analysis 77, 1181-1212 (1996)

8. Gergely, G., Watson, J.: Early social-emotional development: Contingency perception and the social biofeedback model. In: Early social cognition, pp. 101-136. Erlbaum, Hillsdale (1999)

9. Grossberg, S., Merrill, J.W.L.: The hippocampus and cerebellum in adaptively timed learning, recognition, and movement. Journal of Cognitive Neuroscience 8, 257-277 (1996)

10. Lopresti-Goodman, S.M., Richardson, M.J., Silva, P.L., Schmidt, R.C.: Period basin of entrainment for unintentional visual coordination. Journal of Motor Behavior 40(1), 3-10 (2008)

11. Murray, L., Trevarthen, C.: Emotional regulation of interactions vetween twomonth-olds and their mothers. Social perception in infants, 101-125 (1985)

12. Nadel, J.: Imitation et communication entre jeunes enfants. Presse Universitaire de France, Paris (1986)

13. Nadel, J.: The functionnal use of imitation in preverbal infants and nonverbal children with autism. In: Meltzoff, A., Prinz, W. (eds.) The Imitative Mind: Developement, Evolution and Brain Bases. Cambridge University Press, Cambridge (2000)

14. Nadel, J.: Imitation and imitation recognition: their functional role in preverbal infants and nonverbal children with autism, pp. 42-62. Cambridge University Press, UK (2002)

15. Nadel, J., Camaioni, L.: New Perspectives in Early Communicative Development. Routledge, London (1993)

16. Nadel, J., Guerini, C., Peze, A., Rivet, C.: The evolving nature of imitation as a format for communication. In: Nadel, G., Butterworth, J. (eds.) Imitation in Infancy, pp. 209-234. Cambridge University Press, Cambridge (1999)

17. Nadel, J., Prepin, K., Okanda, M.: Experiencing contigency and agency: first step toward self-understanding? In: Hauf, P. (ed.) Making Minds II: Special issue of Interaction Studies 6:3 2005, pp. 447-462. John Benjamins publishing company, Amsterdam (2005)

18. Nadel, J., Tremblay-Leveau, H.: Early social cognition, chapter Early perception of social contingencies and interpersonal intentionality: dyadic and triadic paradigms, pp. 189-212. Lawrence Erlbaum Associates, Mahwah (1999)

19. Oullier, O., de Guzman, G.C., Jantzen, K.J., Lagarde, J., Kelso, J.A.S.: Social coordination dynamics: Measuring human bonding. Social Neuroscience 3(2), 178 $192(2008)$

20. Oullier, O., Kelso, J.A.S.: Coordination from the perspective of Social Coordination Dynamics. In: Encyclopedia of Complexity and Systems Science. Springer, Heidelberg (2009)

21. Pikovsky, A., Rosenblum, M., Kurths, J.: Synchronization: A Universal Concept in Nonlinear Sciences. Cambridge University Press, Cambridge (1981)

22. Pineda, J.A.: The functional significance of mu rhythms: Translating "seeing" and "hearing" into "doing". Brain Research Reviews 50, 57-68 (2005)

23. Prepin, K., Revel, A.: Human-machine interaction as a model of machinemachine interaction: how to make machines interact as humans do. Advanced Robotics 21(15), 1709-1723 (2007)

24. Revel, A.: Contrôle d'un robot mobile autonome par approche neuro-mimétique. Doctorat de traitement de l'image et du signal, Université de Cergy-Pontoise (Novembre 1997) 
25. Richardson, M.J., Marsh, K.L., Isenhower, R.W., Goodman, J.R.L., Schmidt, R.C.: Rodking together: Dynamics of intentional and unitentional interpersonal coordination. Human Movement Science 26, 867-891 (2007)

26. Richardson, M.J., Marsh, K.L., Schmidt, R.C.: Effects of visual and verbal interaction on unintentional interpersonal coordination. Journal of Experimental Psychology: Human Perception and Performance 31(1), 62-79 (2005)

27. Soussignan, R., Nadel, J., Canet, P., Girardin, P.: Sensitivity to social contingency and positive emotion in 2-month-olds. Infancy 10(2), 123-144 (2006)

28. Tognoli, E., Lagarde, J., DeGuzman, G.C., Kelso, J.A.S.: The phi complex as a neuromarker of human social coordination (2007)

29. Tronick, E., Als, H., Adamson, L., Wise, S., Brazelton, T.B.: The infants' response to entrapment between contradictory messages in face-to-face interactions. Journal of the American Academy of Child Psychiatry (Psychiatrics) 17, 1-13 (1978) 